\title{
¿El reflujo gastroesofágico y la aspiración de contenido gástrico están implicados en la etiopatogenia y la progresión de la fibrosis pulmonar idiopática?
}

\author{
FERNANDO TIRAPEGUI S.*, ORLANDO DÍAZ P.* y FERNANDO SALDÍAS P.*
}

Does gastroesophageal reflux and gastric content aspiration have some role in the pathogenesis and progression of Idiopathic Pulmonary Fibrosis?

Gastroesophageal reflux (GERD) and hidden aspiration of gastric contents are probably involved in the pathogenesis and progression of idiopathic pulmonary fibrosis (IPF). The pathological mechanisms involved are decreased pulmonary distensibility and consequent increase of intrathoracic negative pressure during inspiration, as well as decreased control mechanisms of esophageal motility or lower esophageal sphincter. The prevalence of GERD and oesophageal dysmotility was higher in patients with IPF as compared with general population. Among patients with IPF, 67-76\% demonstrated abnormal oesophageal acid exposure. However, no relationship was demonstrated between severity of GERD and severity of IPF. Data are scant on outcomes of antireflux treatment in patients with IPF. Actually, some data suggests that antacid treatment may be deleterious in some IPF patients. After analyzing all the relevant evidence found to date, a causal relationship between GERD, gastric content aspiration and IPF pathogenesis cannot be established. There is scant evidence examining antireflux treatment in idiopathic pulmonary fibrosis patients.

Key words: Gastroesophageal reflux disease, idiopathic pulmonary fibrosis, Respiratory Aspiration of Gastric Contents Antiacids; Esophageal Motility Disorders.

\section{Resumen}

El reflujo gastroesofágico (RGE) y la aspiración oculta de contenido digestivo están probablemente implicados en la etiopatogenia y progresión de la fibrosis pulmonar idiopática (FPI). Los mecanismos patogénicos involucrados son la disminución de la distensibilidad pulmonar y el consiguiente aumento de la presión negativa intratorácica durante la inspiración, así como la disminución de los mecanismos de control de la motilidad esofágica o del tono del esfínter esofágico inferior. La prevalencia de RGE y anomalías de la motilidad esofágica están aumentadas en los pacientes con FPI comparado con la población general. Entre los pacientes con FPI, el 67-76\% demostraron exposición anormal al contenido ácido en el esófago. Sin embargo, no hubo relación entre la gravedad del $R G E$ y la gravedad de la FPI. Los estudios que han examinado el tratamiento antirreflujo en esta población han sido escasos. Incluso, algunos datos sugieren que el tratamiento antiácido puede ser perjudicial en algunos pacientes con esta condición. Después de analizar toda la evidencia relevante encontrada hasta la fecha, concluimos que no se puede establecer una relación causal entre el RGE, la aspiración del contenido gástrico y la patogénesis de la FPI. Además, existe escasa evidencia clínica que haya examinado el tratamiento antirreflujo en pacientes con fibrosis pulmonar idiopática.

Palabras clave: Reflujo gastroesofágico, fibrosis pulmonar idiopática, aspiración respiratoria de contenido gástrico, antiácidos, trastornos de motilidad esofágica.

* Departamento de Enfermedades Respiratorias, Facultad de Medicina, Pontificia Universidad Católica de Chile. 


\section{Introducción}

La Fibrosis Pulmonar Idiopática (FPI), es la forma más común de neumonía intersticial de causa desconocida, caracterizada por un proceso progresivo de fibrosis limitado al pulmón, ocurre principalmente en adultos mayores y se asocia con un patrón histopatológico y radiológico de neumonía intersticial usual (UIP) ${ }^{1}$. El diagnóstico de esta entidad clínica requiere la exclusión de otras formas de neumonía intersticial de causa conocida, especialmente asociadas con la exposición a inhalantes en el ambiente ocupacional, medicamentos o enfermedades sistémicas. Aunque la sobrevida media es 2,5-3,5 años desde el momento del diagnóstico, existe una gran variabilidad en la evolución de la enfermedad de un paciente a otro ${ }^{1}$. Por lo tanto, predecir la evolución de la enfermedad pulmonar en el paciente particular constituye un gran desafío. Se han logrado avances importantes en los últimos años, pudiendo identificar a los pacientes con mayor riesgo de progresión de la enfermedad que podrían beneficiarse de las intervenciones de tratamiento o uso de fármacos antifibró$\operatorname{ticos}^{2}$. Se han identificado fenotipos clínicos con diferente pronóstico y supervivencia. En general, los principales predictores de riesgo o mal pronóstico identificados son la edad avanzada (mayores de 70 años), antecedentes de tabaquismo, índice de masa corporal reducido, trastorno funcional respiratorio severo, la extensión radiológica de la enfermedad y la presencia de hipertensión pulmonar ${ }^{1,2}$.

Desde hace muchos años se ha asumido, con poca evidencia, que la aspiración oculta de contenido gástrico puede representar un mecanismo importante en la etiopatogenia y la exacerbación aguda (EA) de la fibrosis pulmonar idiopática ${ }^{3-5}$. Esta hipótesis se basa en la aparición de daño alveolar difuso en biopsias pulmonares de modelos experimentales y en necropsias de pacientes fallecidos por aspiración de contenido gástrico ${ }^{3}$. El papel potencial del reflujo gastroesofágico (RGE) en la patogénesis o la progresión de la enfermedad ha sido un foco importante de investigación, planteándose un enfoque terapéutico activo y efectivo para el manejo del paciente. En la última guía clínica internacional sobre el tratamiento de la FPI se intentó abordar el papel de la terapia antiácida en el tratamiento de esta condición, siendo definida una recomendación condicional para el uso de la terapia antiácida en el tratamiento de los pacientes con FPI ${ }^{1}$.

\section{Pregunta clínica}

¿El reflujo gastroesofágico y la aspiración de contenido gástrico tienen alguna relación con la etiopatogenia y progresión de la Fibrosis Pulmonar Idiopática?

\section{Métodos}

Se realizó una búsqueda de los estudios relevantes y revisiones sistemáticas en PubMed, Scielo, Epistemonikos, Cochrane Library y Web of Science entre enero de 1980 y marzo de 2018. Las palabras clave de búsqueda y términos $\mathrm{MeSH}$ fueron gastro-oesophageal reflux, heartburn or pirosis, interstitial lung diseases, acute exacerbation, pulmonary fibrosis, interstitial pneumonia and usual interstitial pneumonitis.

El proceso de búsqueda y selección de artículos fue realizado por dos investigadores (FT, FS) de forma independiente y las discordancias fueron discutidas con un tercer investigador (OD), con el propósito de evitar sesgos, dar solución a las discrepancias y a la falta de información. Los artículos primarios y las revisiones sistemáticas fueron evaluados por resúmenes y título, excluyendo aquellos que no cumpliesen los criterios definidos en la pregunta clínica. El proceso de codificación y vaciamiento de información se realizó en una planilla ad hoc que contenía la información de los autores, título del estudio, fecha de publicación, diseño del estudio, fuentes de información, características de la población examinada, características de la intervención, criterios de inclusión y exclusión, estrategias de búsqueda, proceso de selección y recolección de la información, instrumentos empleados para la medición de los resultados, descripción de los resultados primarios y secundarios, síntesis de los resultados y evaluación de los riesgos de sesgo. Para ello se empleó la metodología propuesta por la organización Epistemonikos y la lista de verificación PRISMA-P recomendada para el reporte de la evidencia en una revisión sistemática ${ }^{6}$. En este proceso, se identificaron 319 publicaciones y 28 de ellas cumplieron con los criterios de la revisión.

\section{Revisión de la evidencia}

Los estudios clínicos han demostrado una elevada incidencia de reflujo gastroesofágico (RGE) en pacientes con Fibrosis Pulmonar Idiopática $(67-76 \%)^{7-10}$, estimándose en la población ge- 
neral una prevalencia de RGE sintomático entre el $10-20 \%$ en América del Norte y Europa, con una menor prevalencia en Asia. Los autores han propuesto que, en estos casos, el RGE puede ser causado por la disminución de la distensibilidad pulmonar, como resultado de la infiltración de tejido conectivo en el parénquima pulmonar y el aumento consecuente de la presión negativa intratorácica durante la inspiración ${ }^{11,12}$. Sin embargo, se ha observado que solo un $50 \%$ de los pacientes con FPI refieren los síntomas clásicos de RGE como pirosis, dispepsia y regurgitación, lo que sugiere que los hallazgos clínicos por sí solos son insuficientes para confirmar el diagnóstico y se requieren exámenes complementarios, tales como la manometría esofágica, medición de $\mathrm{pH}$ esofágico de $24 \mathrm{~h}$ e impedanciometría esofági$\mathrm{ca}^{7-10}$. Por esta razón, es necesario enfatizar la importancia de las evaluaciones objetivas para determinar la presencia de RGE en esta población. Se ha sugerido que los eventos de reflujo proximal pueden causar aspiración $\mathrm{y}$, de esta forma, potencialmente conducir al desarrollo de FPI y/o exacerbaciones agudas de esta entidad

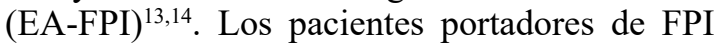
pueden presentar episodios frecuentes de reflujo proximal en ausencia de síntomas $(20 \mathrm{~cm}$ proximal al esfínter esofágico inferior $[\mathrm{EEI}])^{9,15}$. La exposición proximal a ácido ha sido observada con frecuencia en pacientes con esta condición en comparación con sujetos controles asintomáticos y sanos, en quienes los eventos de reflujo faringo-laríngeo (RFL) y de reflujo de columna completa o reflujo $2 \mathrm{~cm}$ distal al esfínter esofágico superior [EES]) son extremadamente $\operatorname{raros}^{16}$. Si se tiene en cuenta que tan solo tres episodios de RFL a la semana pueden causar grave daño anatómico e inflamación laríngea ${ }^{17}$, es posible que la exposición proximal a contenido gástrico pueda provocar severas consecuencias en la vía aérea de estos pacientes.

Hershcovici y cols ${ }^{18}$ realizaron una revisión sistemática examinando la relación entre el reflujo gastroesofágico y las enfermedades pulmonares intersticiales, identificando catorce artículos relacionados con RGE en pacientes con FPI, reportando una elevada prevalencia de RGE en esta entidad clínica; sin embargo, no hubo relación entre la gravedad de ambas condiciones y los resultados obtenidos con las medidas antirreflujo han sido controversiales (Tabla 1). Diez estudios examinaron la prevalencia de RGE en pacientes con FPI empleando evaluación de síntomas, monitorización de $24 \mathrm{~h}$ de $\mathrm{pH}$ esofágico, manometría esofágica o estudio de imágenes. Cuatro estudios examinaron la relación entre RGE o su tratamien- to con la gravedad de la enfermedad pulmonar intersticial. Ninguno de los estudios mencionados fue diseñado para evaluar la relación causal entre RGE y FPI.

Por desgracia, ninguno de los fármacos supresores de ácido cumple la función de barrera del EEI o elimina el reflujo o el riesgo de aspiración, por lo que su papel como parte del manejo de la FPI es aún incierto. En un estudio clásico, Lee y $\operatorname{cols}^{19}$ midieron los niveles de pepsina en 24 pacientes con EA-FPI comparándolo con 30 pacientes control con FPI en fase estable. Los autores encontraron concentraciones medibles de pepsina en el lavado broncoalveolar (LBA) en la mayoría de los pacientes con FPI en fase estable, lo que sugiere que la aspiración oculta es común en ellos. Pero, más importante aún, observaron que un $33 \%$ de los pacientes con EA-FPI tenían concentraciones elevadas de pepsina en el LBA, lo que apunta a que la aspiración oculta podría estar involucrada en algunos casos en la etiopatogenia y progresión de la enfermedad. En la misma línea, Tcherakian y cols ${ }^{20}$ observaron una mayor asociación entre RGE y EA-FPI en pacientes con FPI asimétrica. La mayoría de las exacerbaciones de la enfermedad acontecieron en el pulmón con mayor daño anatómico, lo que correspondía al hemitórax ubicado en posición dependiente al dormir.

Un análisis post hoc de las ramas placebo de tres estudios clínicos, correspondiendo a 242 pacientes, evidenció que la EA-FPI ocurrió solo en sujetos que no estaban recibiendo terapia supresora de ácido (inhibidores de la bomba de protones [IBP] o antagonistas $\mathrm{H}_{2}$ ), atribuyéndole un potencial efecto protector en la injuria pulmonar por microaspiración ${ }^{21}$. Tanto es así, que el $51 \%$ de los pacientes que empleaba terapia supresora de ácido al inicio del estudio mostró una menor reducción de la capacidad vital forzada (CVF) a las 30 semanas de seguimiento, lo que sugiere un efecto benéfico en estos pacientes, y le confiere al RGE un papel significativo en la progresión de la enfermedad ${ }^{22}$.

Sin embargo, en contraposición a estos resultados, el análisis post hoc de los estudios INPULSIS han demostrado que la incidencia de EA-FPI en el grupo que empleaba terapia supresora de ácido al inicio del estudio fue superior comparado con aquellos que no fueron tratados: 33 (47,8\%) versus $373(37,6 \%)$ casos, hazard ratio: $1,78$ (IC95\% 0,92-3,43 $)^{22}$ y la CVF disminuyó en mayor grado en comparación con quienes no emplearon dicha terapia. Esto sugiere que la terapia supresora de ácido podría ser deletérea en algunos pacientes con esta condición ${ }^{23}$. La razón para 


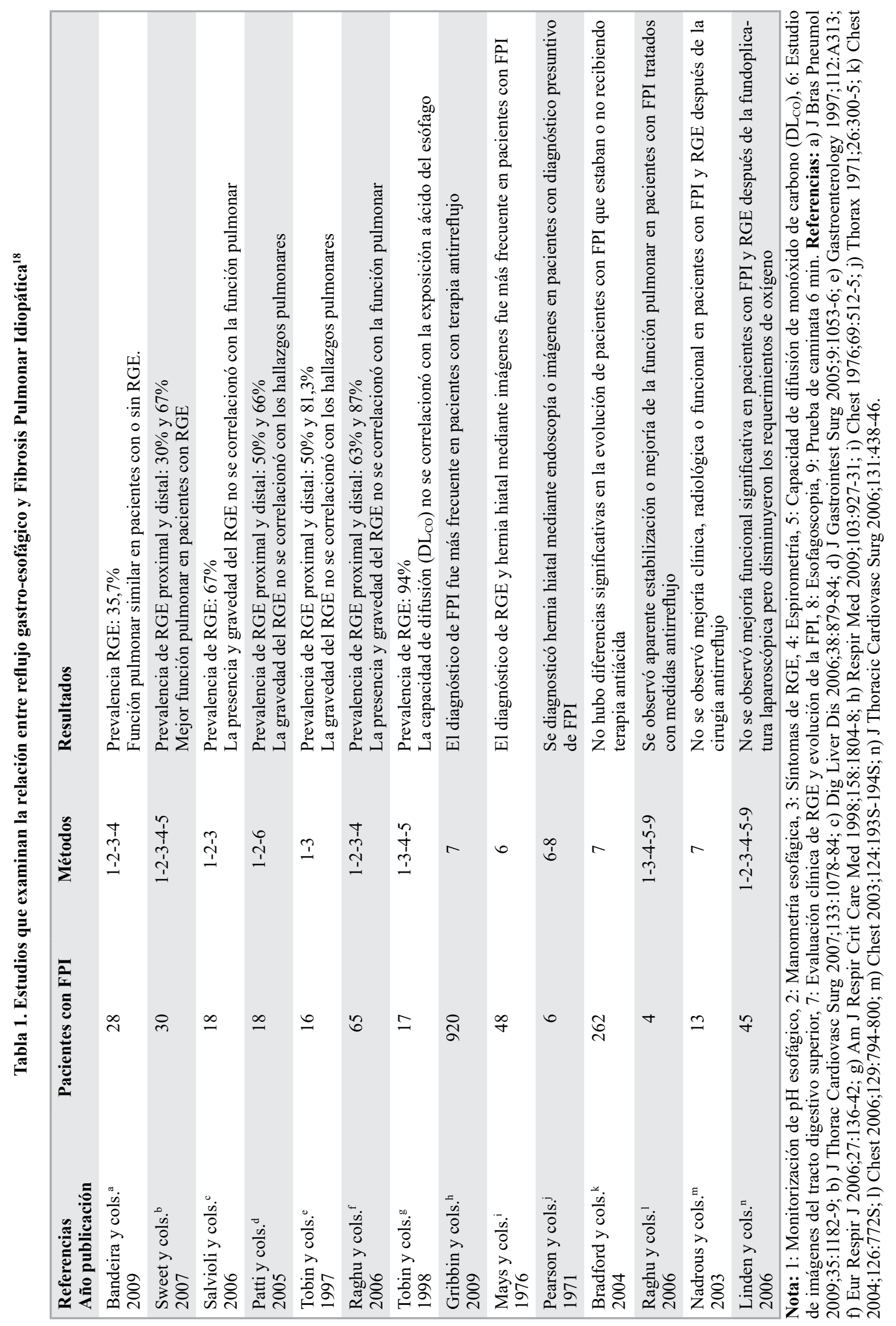


estos resultados contradictorios no está clara, se ha especulado que podría deberse a la persistencia de RGE a pesar de la terapia supresora, o quizás al daño inducido por reflujo alcalino. Kreuters y cols han intentado abordar más a fondo el papel de la terapia supresora de ácido en pacientes con FPI mediante el análisis de los datos agrupados de los grupos placebo de tres estudios clínicos con Pirfenidona (CAPACITY 004, CAPACITY 006 y ASCEND) ${ }^{24}$. Estos estudios incluyeron 624 pacientes con y sin terapia supresora de ácido en el momento de enrolamiento, los análisis no mostraron ningún beneficio de la terapia supresora de ácido en múltiples desenlaces evaluados, incluyendo un desenlace compuesto, que incluía la progresión de la enfermedad, el cambio de la CVF y la mortalidad ${ }^{24}$.

El problema es aún más complejo debido a la posible interacción entre la terapia antiácida y los medicamentos antifibróticos (Pirfenidona o Nintedanib). Por ejemplo, algunos IBP como el Omeprazol son inductores moderados de la enzima CYP1A2 y su uso concomitante con Pirfenidona puede reducir, al menos teóricamente, los niveles plasmáticos del fármaco antifibróti$\mathrm{co}^{25}$. Por otro lado, la terapia supresora de ácido no es suficiente para controlar el RGE ácido en muchos pacientes, no controla el reflujo alcalino y no previene la microaspiración ${ }^{26}$. Solamente la cirugía antirreflujo, que incluye la reparación de la hernia hiatal y la fundoplicatura, por lo general mediante laparoscopia, tiene el potencial de controlar completamente el RGE y disminuir el riesgo de broncoaspiración ${ }^{27}$.

Ghebre y Raghu en una reciente revisión sugieren que la terapia antiácida (por ejemplo, uso de IBP) puede jugar un papel beneficioso en la evolución de los pacientes con FPI a pesar de su incapacidad para controlar el reflujo gástrico per se o la microaspiración ${ }^{28}$. Los mecanismos alternativos y biológicamente plausibles que pueden subyacer al efecto beneficioso de los IBP en esta entidad pueden incluir la reducción de las moléculas fibroinflamatorias, la regulación positiva de los mecanismos citoprotectores, la inhibición de la proliferación de fibroblastos y la supresión de la acidez gástrica ${ }^{29-31}$. Sin embargo, es importante reconocer que existen varios efectos secundarios no fatales asociados con el uso intermitente $\mathrm{y} / \mathrm{o}$ prolongado de fármacos IBP, que incluyen la erupción cutánea, fatiga, diarrea, dolor de cabeza, reaparición de los síntomas y regurgitación ácida después de la interrupción del tratamiento, aumento de los eventos de reflujo no ácido, riesgo de osteoporosis, mayor riesgo de neumonía adquirida en la comunidad, deterioro cognitivo y eventos cardiovasculares adversos mayores. Otro posible efecto adverso de los fármacos IBP es la alteración de la homeostasis del microbioma gástrico y esofágico con estudios que han encontrado una asociación entre una mayor abundancia de secuencias bacterianas específicas o cargas bacterianas y la progresión de la enfermedad fibrótica, incluida la acelerada caída de la capacidad vital forzada.

Con la evidencia clínica disponible, no podemos recomendar un algoritmo de detección, diagnóstico y/o tratamiento específico para la microaspiración en pacientes con fibrosis pulmonar idiopática ${ }^{2,47-13}$. Mucho se ha escrito sobre la necesidad de pesquisar y tratar el reflujo gastroesofágico en esta población. Sin embargo, aún existe controversia en la literatura médica acerca del estudio diagnóstico y tratamiento sistemático de esta condición ${ }^{18,21-24}$. Esto se debe principalmente a que no existen datos concluyentes que demuestren beneficio clínico con el tratamiento del reflujo gastroesofágico en los pacientes con fibrosis pulmonar idiopática y existen riesgos asociados al tratamiento médico y quirúrgico. Los estudios han comunicado mayor riesgo de neumonía y fractura de cadera en asociación con el uso de inhibidores de la bomba de protones. Además, los IBP solo cambian la acidez del reflujo; no previenen el reflujo o la microaspiración del contenido gástrico.

Esperemos que los estudios clínicos en curso "Pilot Trial Of Omeprazole in Idiopathic Pulmonary Fibrosis (IPF)" (PPIPF; NCT02085018) ${ }^{32}$ y "Treatment of IPF With Laparoscopic Anti-Reflux Surgery" (WRAP-IPF; NCT01982968) 33 nos proporcionen más luces sobre su importancia en la etiopatogenia, el manejo y la evolución de los pacientes con esta condición clínica.

\section{Conclusiones}

Después de analizar la evidencia clínica encontrada hasta la actualidad, no está claro si la elevada prevalencia de reflujo gastroesofágico en pacientes con FPI está relacionada patológicamente con el desarrollo, la progresión o la exacerbación de la enfermedad a lo largo del tiempo. Tampoco está claro si el tratamiento antiácido es una terapia efectiva para manejar esta condición, independientemente de la presencia de RGE subyacente. En presencia de RGE anormal, los estudios en curso examinarán el abordaje terapéutico óptimo y si las terapias antiácidas son adecuadas o si los tratamientos definitivos, como la fundoplicatura de Nissen están justificados y, de ser así, 
en qué grupo específico de pacientes con FPI. Las recomendaciones de las guías clínicas internacionales se basan en la escasa evidencia clínica y experimental disponible con las limitaciones inherentes que conlleva el estudio de este problema. En algunos contextos, estos procesos podrían dar lugar a recomendaciones controvertidas que tienen efectos importantes en la atención clínica. En virtud de los resultados de la evidencia clínica publicada hasta la fecha, estudios de calidad moderada y baja, se sugiere que en algunos pacientes con Fibrosis Pulmonar Idiopática la aspiración podría determinar la progresión o gatillar exacerbaciones de la enfermedad, pero que la mayoría de las EA-FPI no estarían relacionadas con este fenómeno.

\section{Bibliografía}

1.- RAGHU G, ROCHWERG B, ZHANG Y, GARCÍA CA, AZUMA A, BEHR J, et al. American Thoracic Society; European Respiratory society; Japanese Respiratory Society; Latin American Thoracic Association. An Official ATS/ERS/JRS/ALAT Clinical Practice Guideline: Treatment of Idiopathic Pulmonary Fibrosis. An Update of the 2011 Clinical Practice Guideline. Am J Respir Crit Care Med 2015; 192: e3-19.

2.- KING TE, PARDO A, SELMAN M. Idiopathic pulmonary fibrosis. Lancet 2011; 378: 1949-61.

3.- WARE LB, MATTHAY MA. The acute respiratory distress syndrome. N Engl J Med 2000; 342: 1334-49.

4.- LEE JS, COLLARD HR, RAGHU G, SWEET MP, HAYS SR, CAMPOS GM, et al. Does chronic microaspiration cause idiopathic pulmonary fibrosis? Am J Med 2010; 123: 304-11.

5.- COLLARD HR, MOORE BB, FLAHERTY KR, BROWN KK, KANER RJ, KING TE Jr, et al. Acute exacerbations of idiopathic pulmonary fibrosis. Am J Respir Crit Care Med 2007; 176: 636-43.

6.- SHAMSEER L, MOHER D, CLARKE M, GHERSI D, LIBERATI A, PETTICREW M, SHEKELLE P, STEWART L A; PRISMA-P Group. Preferred reporting items for systematic review and meta-analysis protocols (PRISMA-P) 2015: elaboration and explanation. BMJ 2015; 350: g7647.

7.- RAGHU G, FREUDENBERGER TD, YANG S, CURTIS JR, SPADA C, HAYES J, et al. High prevalence of abnormal acid gastro-oesophageal reflux in idiopathic pulmonary fibrosis. Eur Respir J 2006; 27: 136-42.

8.- TOBIN RW, POPE CE $2^{\text {nd }}$, PELLEGRINI CA, EMOND MJ, SILLERY J, RAGHU G. Increased prevalence of gastroesophageal reflux in patients with idiopathic pulmonary fibrosis. Am J Respir Crit Care Med 1998; 158 : 1804-8.

9.- SWEET MP, PATTI M G, LEARD LE, GOLDEN JA, HAYS SR, HOOPES C, et al. Gastroesophageal reflux in patients with idiopathic pulmonary fibrosis referred for lung transplantation. J Thorac Cardiovasc Surg 2007; 133: 1078-84.

10.- BANDEIRA CD, RUBIN AS, CARDOSO PF, MOREIRA JDAS, MACHADO MDA M. Prevalence of gastroesophageal reflux disease in patients with idiopathic pulmonary fibrosis. J Bras Pneumol 2009; 35: 1182-9.

11.- DEMETER P, PAP A. The relationship between gastroesophageal reflux disease and obstructive sleep apnea. J Gastroenterol 2004; 39: 815-20.

12.- RAGHU G, YANG ST, SPADA C, HAYES J, PELLEGRINI CA. Sole treatment of acid gastroesophageal reflux in idiopathic pulmonary fibrosis: a case series. Chest 2006; 129: 794-800.

13.- FAHIM A, CROOKS M, HART SP. Gastroesophageal reflux and idiopathic pulmonary fibrosis: a review. Pulm Med 2011; 2011: 634613. doi: 10.1155/2011/634613.

14.- PATTI MG, DEBAS HT, PELLEGRINI CA. Clinical and functional characterization of high gastroesophageal reflux. Am J Surg 1993; 165: 163-6.

15.- D'OVIDIO F, SINGER LG, HADJILIADIS D, PIERRE A, WADDELL TK, DE PERROT M, et al. Prevalence of gastroesophageal reflux in end-stage lung disease candidates for lung transplant. Ann Thorac Surg 2005; 80: $1254-60$.

16.- HOPPO T, SANZ AF, NASON KS, CARROLL T L, ROSEN C, NORMOLLE DP, et al. How much pharyngeal exposure is "normal"? Normative data for laryngopharyngeal reflux events using hypopharyngeal multichannel intraluminal impedance (HMII). J Gastrointest Surg 2012; 16: 16-24.

17.- KOUFMAN JA. The otolaryngologic manifestations of gastroesophageal reflux disease (GERD): A clinical investigation of 225 patients using ambulatory 24-hour $\mathrm{pH}$ monitoring and an experimental investigation of the role of acid and pepsin in the development of laryngeal injury. Laryngoscope 1991; 101 (4 Pt 2 Suppl 53): 1-78.

18.- HERSHCOVICI T, JHA LK, JOHNSON T, GERSON L, STAVE C, MALO J, et al. Systematic review: the relationship between interstitial lung diseases and gastro-oesophageal reflux disease. Aliment Pharmacol Ther 2011; 34: 1295-305.

19.- LEE JS, SONG JW, WOLTERS PJ, ELICKER BM, KING TE JR, KIM DS, et al. Bronchoalveolar lavage pepsin in acute exacerbation of idiopathic pulmonary fibrosis. Eur Respir J 2012; 39: 352-8.

20.- TCHERAKIAN C, COTTIN V, BRILLET PY, FREYNET O, NAGGARA N, CARTON Z, et al. Progression of idiopathic pulmonary fibrosis: lessons from asymmetrical disease. Thorax 2011; 66: 226-31.

21.- LEE JS, COLLARD HR, ANSTROM KJ, MARTÍNEZ FJ, NOTH I, ROBERTS RS, et al. Anti-acid treatment and disease progression in idiopathic pulmonary fibrosis: an analysis of data from three randomised controlled trials. Lancet Respir Med 2013; 1: 369-76. 
22.- COLLARD HR, RICHELDI L, KIM DS, TANIGUCHI H, TSCHOEPE I, LUISETTI M, et al. Acute exacerbations in the INPULSIS trials of nintedanib in idiopathic pulmonary fibrosis. Eur Respir J 2017; 49 (5) pii: 1601339. doi 10.1183/13993003.01339-2016.

23.- RAGHU G, CRESTANI B, BAILES Z, et al. Effect of anti-acid medication on reduction in FVC decline with nintedanib. Eur Respir J 2015; 46 (Suppl 59): OA4502.

24.- KREUTER M, WUYTS W, RENZONI E, KOSCHEL D, MAHER TM, KOLB M, et al. Antiacid therapy and disease outcomes in idiopathic pulmonary fibrosis: a pooled analysis. Lancet Respir Med 2016; 4: 381-9

25.- EUROPEAN MEDICINES AGENCY. Esbriet product information. www.ema.europa.eu/docs/en_GB/ document_library/EPAR_Poduct_Information/human/002154/WC500103049.pdf. (Date last accessed: march 12, 2018).

26.- RAGHU G, MEYER KC. Silent gastrooesophageal reflux and microaspiration in IPF: mounting evidence for anti-reflux therapy? Eur Respir J 2012; 39: 242-5.

27.- RAGHU G, MORROW E, COLLINS BF, HO LA, HINOJOSA MW, HAYES JM, et al. Laparoscopic anti-reflux surgery for idiopathic pulmonary fibrosis at a single centre. Eur Respir J 2016; 48: 826-32.

28.- GHEBRE YT, RAGHU G. Idiopathic Pulmonary Fibrosis: Novel concepts of proton pump inhibitors as antifibrotic drug. Am J Respir Crit Care Med 2016; 193 : 1345-52.

29.- YOSHIDA N, YOSHIKAWA T, TANAKA Y, FUJITA N, KASSAI K, NAITO Y, et al. A new mechanism for anti-inflammatory actions of proton pump inhibitors-inhibitory effects on neutrophil-endothelial cell interactions. Aliment Pharmacol Ther 2000; 14 (Suppl 1): 74-81.

30.- KEDIKA RR, SOUZA RF, SPECHLER SJ. Potential anti-inflammatory effects of proton pump inhibitors: a review and discussion of the clinical implications. Dig Dis Sci 2009; 54: 2312-7.

31.- GHEBREMARIAM YT, COOKE JP, GERHART W, GRIEGO C, BROWER JB, DOYLE-EISELE M, et al. Pleiotropic effect of the proton pump inhibitor esomeprazole leading to suppression of lung inflammation and fibrosis. J Transl Med 2015; 13: 249.

32.- www.clinicaltrials.gov. Pilot Trial Of Omeprazole in Idiopathic Pulmonary Fibrosis (IPF) (PPIPF). https:// clinicaltrials.gov/ct2/ show/NCT02085018. Date last accessed: October 11, 2017. Clinical Trials identifier: NCT02085018

33.- www.clinicaltrials.gov. Treatment of IPF with laparoscopic anti-reflux surgery (WRAP-IPF). https:// clinicaltrials.gov/ct2/ show/NCT01982968. Date last accessed: October 11, 2017. Clinical Trials identifier: NCT01982968.
Correspondencia a:

Dr. Fernando Tirapegui Sanhueza

Departamento de Enfermedades Respiratorias, Facultad de Medicina, Pontificia Universidad Católica de Chile. Diagonal Paraguay 362, Sexto Piso, Santiago, Chile.

Email: ftirapegui@uc.cl 